\title{
Prolonged Response to Anti-PD-1 Antibody Therapy in Chemotherapy-Refractory Cholangiocarcinoma With High Tumor Mutational Burden
}

\author{
Olumide Gbolahan, MBBS ${ }^{a}$; Neda Hashemi-Sadraei, MBBSb; and Bert O’Neil, MD ${ }^{c}$
}

\section{ABSTRACT}

Management of advanced intrahepatic cholangiocarcinoma (iCCA) is challenging and overall survival is poor. Progress in the development of new therapeutic options for metastatic cholangiocarcinoma (CCA) has been slow; hence, to date, there are no approved secondline agents in this setting. Although the development of immune checkpoint inhibitors has significantly improved overall survival in a variety of malignancies, there has not been a clinically important impact in CCA. This report presents a 66-year-old patient with chemotherapy-refractory iCCA who experienced a prolonged response to immunotherapy. Tumor genome profiling revealed a high tumor mutation burden of 17 mutations per megabase in the absence of microsatellite instability. He was started on immunotherapy with nivolumab and has experienced an ongoing response for 16 months without clinical symptoms and only minimal radiologic disease.

J Natl Compr Canc Netw 2019;17(6):644-648 doi: $10.6004 /$ jnccn.2019.7304

anniversity of Alabama at Birmingham School of Medicine, Birmingham, Alabama; ${ }^{b}$ University of New Mexico School of Medicine, Albuquerque, New Mexico; and Indiana University School of Medicine, Indianapolis, Indiana.
Intrahepatic cholangiocarcinoma (iCCA) remains recalcitrant to treatment, with a median survival of only 26 to 30 months in localized and resectable disease. ${ }^{1,2}$ In the more common locally advanced/metastatic setting, outcomes are even more dire. A median overall survival of 11.7 months was reported with combination gemcitabine + cisplatin in the first-line setting. ${ }^{3}$ Despite this high mortality and reports of increasing incidence of iCCA, ${ }^{4}$ little progress has been made in the development of new therapeutic options. Indeed, there are no agents (single-therapy or combination) approved in the second line for advanced/metastatic iCCA, and in this era of precision medicine and immune therapy, there are no biologic agents used outside clinical trials. Preliminary results of the KEYNOTE-028 study (ClinicalTrials.gov Identifier: NCT02054806) showed no radiographic complete response among 24 patients with PD-L1-positive $(>1 \%$ expression threshold) advanced biliary tree cancer treated with pembrolizumab. The overall response rate was $17 \%$, and 5 patients remained on treatment for $40+$ to $42+$ weeks at the time of the report. ${ }^{5}$

This report presents a patient with chemotherapyrefractory recurrent metastatic iCCA who has experienced a durable response to third-line anti-PD-1 immune checkpoint inhibitor (ICI) therapy for $>12$ months. Targeted genetic sequencing before treatment revealed an unusually high tumor mutational burden (TMB), and we posit that this is the reason for the prolonged response.

\section{Case Report}

A 66-year-old white man with a history of type 2 diabetes mellitus presented to the emergency room after a nearsyncopal event. He was noted to be jaundiced, and an ultrasound scan of the liver revealed a $2.4-\times 2.0-\mathrm{cm}$ hypoechoic mass in the central right hepatic lobe. This was confirmed by subsequent imaging with CT and MRI. No lymph node involvement or evidence of lesions suspicious for metastasis was evident within the abdomen or thorax. Cytology through fine-needle aspiration of the mass via an endoscopic ultrasound procedure 
showed moderately differentiated cholangiocarcinoma (CCA). The patient underwent exploratory laparotomy, and right hepatectomy, cholecystectomy, extrahepatic bile duct resection, and Roux-en-Y hepaticojejunostomy were performed in June 2015. Within the right hepatectomy specimen, there was a $3.3-\mathrm{cm}$ poorly differentiated CCA with involvement of the vascular (hepatic vein) margin. The mass was staged as poorly differentiated pT2N0 CCA (stage II) after R1 resection; CA 19-9 level was not elevated $(<37 \mathrm{U} / \mathrm{mL})$. The patient received adjuvant chemoradiation with capecitabine and 50.4 Gy of radiation in 28 fractions.

Three months after chemoradiation, CT revealed indeterminate subcentimeter pulmonary nodules. Repeat scan 3 months later showed a 2.2-cm left hepatic lobe mass. No pulmonary involvement was seen. Biopsy results of the lesion in the left hepatic lobe were consistent with metastatic CCA. At that point, the patient received 8 cycles of chemotherapy with gemcitabine + cisplatin. At the end of systemic therapy in July 2016, imaging revealed a partial response in the left hepatic lobe mass, which now measured $1.6 \mathrm{~cm}$, with no other lesions. A PET/CT scan obtained in August 2016 to assess residual tumor activity and rule out more widespread disease before planned local therapy for the liver disease confirmed the liver mass $(2.3 \mathrm{~cm}$; standardized uptake value [SUV], 14.7) and also showed a 1.6-cm hypermetabolic umbilical nodule (SUV, 11.4) and a $1.3-\mathrm{cm}$ perirectal nodule (SUV, 11.7). The patient was started on systemic therapy with second-line therapy with combination 5-fluorouracil/leucovorin/oxaliplatin (FOLFOX) and received palliative stereotactic body radiation therapy to the liver and painful umbilical nodules. Upon further disease progression, he was referred to our phase I clinic.

At his first visit to our clinic, he reported that FOLFOX had recently been stopped in December 2016 after 6 cycles due to clinical (worsening abdominal pain and ascites) and radiologic progression. He reported mild abdominal pain and fatigue, but his ECOG performance status was 1 , with minimal adverse effects from the previous chemotherapy. The right hepatectomy tumor specimen was sent for panel-based tumor genomic profiling on the FoundationOne platform (Foundation Medicine), ${ }^{6}$ which revealed a KRAS (exon 4) A146T mutation and BRCA2 R3052Q mutation (Table 1). Results of PD-L1 staining were negative. Importantly, the testing also found a TMB of 17 mutations/Mb in the absence of microsatellite instability (MSI). On the basis of high $\mathrm{TMB}$, the patient was started on anti-PD-1 antibody therapy (nivolumab) in March 2017. Interval imaging 2 months after initiation of therapy showed a stable $1.5-\mathrm{cm}$ left hepatic lobe nodule, a partial response in the perirectal lesion, and a complete response in previously described inguinal canal nodules. To date, the patient has had minimal residual disease since scans obtained 7 months into treatment (Figure 1). His clinical symptoms (abdominal pain and ascites) have completely resolved, and he has continued to receive nivolumab with minimal adverse events except for grade 1 fatigue.

\section{Discussion}

ICIs (immunotherapy) have transformed the management of a variety of advanced and metastatic solid tumors, particularly melanoma and non-small cell lung cancer (NSCLC). In addition to improving progressionfree and overall survival, immune therapy has the potential to produce very prolonged responses and possibly even cures in patients with previously incurable cancer. ${ }^{7}$ However, the therapeutic benefit of immunotherapy has been inconsistent across the panoply of tumors for which it is used. A number of patients with melanoma or NSCLC have disappointing responses to immunotherapy. There is therefore an ongoing search for biomarkers that predict response to therapy.

PD-L1 expression is the most widely studied biomarker of response to immune therapy and is the most commonly used metric. Higher expression of PD-L1 is associated with improved benefit from immune therapy in a number of cancer types. ${ }^{8}$ However, the limitations of PD-L1 expression as a predictor of response to immune

Table 1. Panel-Based Tumor Genomic Profile of Right Hepatectomy Cholangiocarcinoma Specimen

\begin{tabular}{|lll|}
\hline Key Molecular Alterations & Variants of Unknown Significance & PD-L1 Immunohistochemical Analysis (\%) \\
\hline KRAS A146T & ROS1 K2099N & Tumor score, 0 \\
\hline BRCA2 R3052O & AXL Q361P & Tumor-infiltrating immunocytes, 0 \\
\hline KDM6A R971fs ${ }^{*} 8$ & FGF23 P153A & \\
\hline SPTA1 R250H & CHEK2 A215T & \\
\hline TMB17 mutations/megabase & TSC2 R901C & \\
\hline Microsatellite stable & & \\
\hline
\end{tabular}

${ }^{a}$ Curated list of alterations potentially targetable with FDA-approved drugs from the appendix of variants of unknown significance provided within the FoundationOne tumor genomic profile result. 

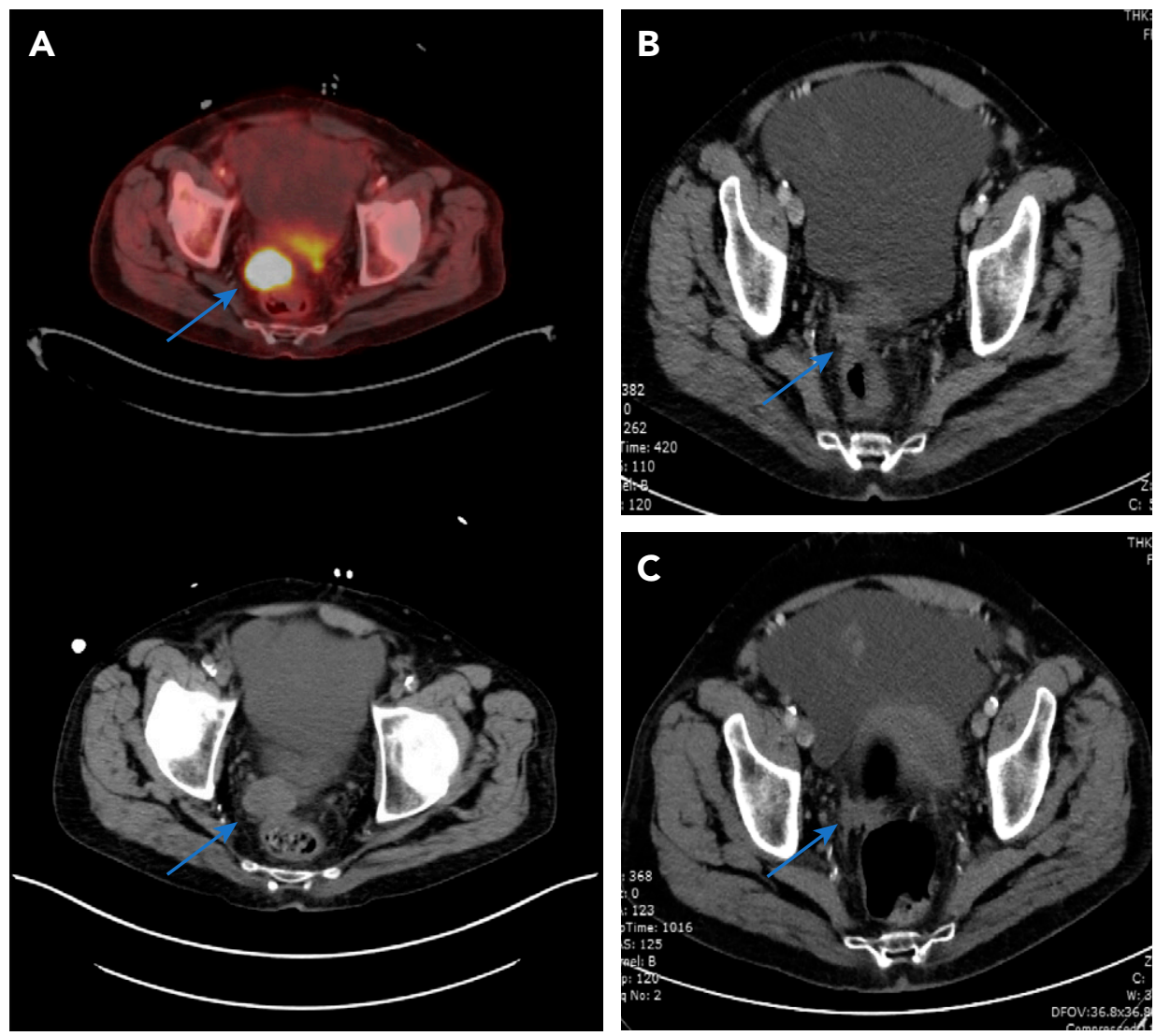

Figure 1. Perirectal metastatic lesion (arrows) responding to nivolumab. (A) Perirectal PET-avid lesion measuring $2.3 \mathrm{~cm}$ at baseline. (B) Perirectal lesion measuring $1.7 \mathrm{~cm} 2$ months after start of nivolumab. (C) Minimal residual perirectal lesion 7 months after start of nivolumab.

therapy have been documented, ${ }^{9,10}$ especially given that responses are also seen in "PD-L1-negative tumors" across tumor types.

Mismatch repair (MMR) deficiency (often defined by MSI) is caused by loss or methylation-based suppression of the genes that encode MMR proteins ( $M L H 1, M S H 2$, MSH6, PMS2, and EpCAM). MMR deficiency is another established predictive marker for response to immune therapy. In a pivotal phase II study (ClinicalTrials.gov identifier: NCT01876511), treatment with pembrolizumab was associated with a response rate of $53 \%$ in MMRdeficient tumors (colorectal and noncolorectal cancers) compared with $0 \%$ for MMR-proficient tumors. ${ }^{11} \mathrm{MMR}$ deficiency leads to a significant increase in mutation frequency and consequently a higher neoantigen burden of the tumor. This increase in neoantigen quantity and diversity is thought to stimulate an improved immune response to the tumor. ${ }^{12}$ In the absence of MMR deficiency, an increase in TMB also seems to be an independent and bona fide predictive biomarker of response to ICI therapy..$^{13}$ For example, in melanoma, Snyder et $\mathrm{al}^{14}$ showed that presence of $>100$ nonsynonymous mutations per exome was associated with an improved response rate and survival in response to anti-CTLA-4 antibody therapy. Similar findings have been described with anti-PD-1 therapy in NSCLC. ${ }^{15}$ For melanoma and lung cancer, TMB may be driven by UV light exposure and chemically driven mechanisms rather than by MMR loss.

Biliary tree cancers are generally characterized by a low TMB, with median TMB of 2.9 to 5.7 mutations/Mb reported. ${ }^{16,17}$ The iCCA in our patient had a TMB of 17 mutations/Mb, which is impressive, given that Salem et $\mathrm{al}^{16}$ reported a high TMB (threshold of 15 mutations/Mb) in only $2.9 \%$ of patients with biliary tract cancer. The high TMB was the motivation for recommending anti-PD-1 therapy to our patient.

Use of TMB as a marker for response to ICI therapy is still evolving. A major challenge is defining a cutoff for low versus high TMB. Although a level of $>20$ mutations $/ \mathrm{Mb}$ is reported in the literature for defining a high TMB, the threshold adopted in clinical practice is usually lower. ${ }^{13,18}$ In a recent examination of the efficacy of combination ipilimumab + nivolumab in NSCLC, a clinically relevant, less restrictive TMB threshold of 10 mutations/Mb was used. ${ }^{19}$ It is also not clear whether this should be a 
dichotomous rather than a continuous classification, and some investigators have defined an intermediate TMB. ${ }^{13}$ Furthermore, a case could be made for individualizing TMB classification based on tumor histology, because a TMB of 10 , which may be considered low in NSCLC, may have a different connotation in pancreatic ductal adenocarcinoma and biliary tree cancer or in other malignancies with generally low tumor mutations. Answering these questions will become important as TMB becomes more widely validated as a prognostic biomarker for immunotherapy.

Interestingly, although next-generation sequencing showed that the CCA was microsatellite stable (MSS), paired tumor and normal tissue immunohistochemistry (IHC) showed loss of MLH1/PMS2 expression in the tumor. Subsequent PCR-based MSI analysis using the Promega kit did not show alterations in any microsatellite locus, consistent with MSS. This was in agreement with next-generation sequencing results. The discordance rate between IHC and PCR for detecting MSI is $<5 \%$, and the performance (and interpretation) of IHC is more prone to errors than PCR-based testing., ${ }^{20,21}$ Importantly, although testing for MSI and MMR proteins is standardized and validated in colorectal and endometrial cancer, this is not the case in other Lynch syndrome-associated malignancies. For example, a review of 834 cases of ovarian cancer reported a concordance rate of only $68 \%$ between IHC- and PCR-based testing. ${ }^{22}$ This suggests that data for colorectal cancer cannot be extrapolated to other malignancies. In our patient, the agreement between PCR and next-generation sequencing increased our confidence that he had MSS disease, although it can be argued that MLH1/PMS2 loss by IHC is sufficient to identify MMR deficiency. Nevertheless, we did not perform any tests to directly assess MLH1 promoter hypermethylation. Investigators have reported MLH1 promoter hypermethylation in approximately $20 \%$ of MSS colorectal cancer cases, although in this series, it did not translate to loss of MLH1 expression. ${ }^{23}$ Furthermore, in a cohort of patients with ovarian cancer, approximately $10 \%$ (3 of 28 samples) of MSS (PCR-based assay) samples had MLH1 promoter hypermethylation. ${ }^{24}$ We therefore concede that high TMB may be related to MMR deficiency secondary to $M L H 1$ alteration. Indeed, in the absence of a high tumor mutational burden, $M L H 1$ loss would be a valid indication for the use of ICI therapy in our patient.

Another interesting hypothesis regarding our patient is whether genomic instability caused by the $B R C A$ mutation (independent of a high TMB) may have contributed to prolonged survival and the durable response to immunotherapy. $B R C A$ mutations are rare in $C C A,{ }^{25,26}$ and the BRCA2 R3052Q mutation detected in our patient was considered a variant of unknown significance. The role of BRCA mutations specifically and homologous recombination deficiency (HRD; marker of genomic instability) in determining response to immunotherapy has not been clearly defined. A few reports link HRD to a high $\mathrm{TMB},{ }^{27,28}$ but data are still early. Because HRD has been explored as a biomarker for the use of PARP inhibitors and may become important for immunotherapy, it is important to develop an assay that would be applicable across malignancies. The HRD assay that is currently available was developed for breast and ovarian cancers.

\section{Conclusions}

This report presents a patient with chemotherapyresistant iCCA and a high TMB who experienced prolonged disease control with immunotherapy. This impressive response highlights the usefulness of tumor genome profile testing as a tool for guiding therapy. These findings also strengthen the case for exploring high TMB as a tumor agnostic biomarker of response to immune therapy. We highlight a potential challenge with the current approach to detecting MSI and the need to validate this approach in other Lynch syndrome-associated malignancies.

Submitted August 9, 2018; accepted for publication March 29, 2019

Disclosures: The authors have disclosed that they have no financial interests, arrangements, or affiliations with the manufacturers of any products discussed in this article or their competitors.

Correspondence: Olumide Gbolahan, MBBS, University of Alabama at Birmingham School of Medicine, NP2540J, 1802 Sixth Avenue South, Birmingham, AL 35233. Email: ogbolahan@uabmc.edu

\section{References}

1. Endo I, Gonen M, Yopp AC, et al. Intrahepatic cholangiocarcinoma: rising frequency, improved survival, and determinants of outcome after resection. Ann Surg 2008;248:84-96.

2. de Jong MC, Nathan $\mathrm{H}$, Sotiropoulos GC, et al. Intrahepatic cholangiocarcinoma: an international multi-institutional analysis of prognostic factors and lymph node assessment. J Clin Oncol 2011;29:3140-3145.

3. Valle J, Wasan H, Palmer DH, et al.. Cisplatin plus gemcitabine versus gemcitabine for biliary tract cancer. N Engl J Med 2010;362: 1273-1281.

4. Saha SK, Zhu AX, Fuchs CS, et al. Forty-year trends in cholangiocarcinoma incidence in the U.S.: intrahepatic disease on the rise. Oncologist 2016; 21:594-599.

5. Bang YJ, Doi T, De Braud F, et al. Safety and efficacy of pembrolizumab (MK-3475) in patients (pts) with advanced biliary tract cancer: interim results of KEYNOTE-028 [abstract]. Eur J Cancer 2015;51(Suppl 3): Abstract 525.

6. Frampton GM, Fichtenholtz A, Otto GA, et al. Development and validation of a clinical cancer genomic profiling test based on massively parallel DNA sequencing. Nat Biotechnol 2013;31: 1023-1031.

7. Schadendorf D, Hodi FS, Robert C, et al. Pooled analysis of long-term survival data from phase II and phase III trials of ipilimumab in unresectable or metastatic melanoma. J Clin Oncol 2015;33: 1889-1894. 
8. Reck M, Rodríguez-Abreu D, Robinson AG, et al.. Pembrolizumab versus chemotherapy for PD-L1-positive non-small-cell lung cancer. N Engl J Med 2016;375:1823-1833.

9. Grigg C, Rizvi NA. PD-L1 biomarker testing for non-small cell lung cancer: truth or fiction? J Immunother Cancer 2016;4:48.

10. McLaughlin J, Han G, Schalper KA, et al. Quantitative assessment of the heterogeneity of PD-L1 expression in non-small-cell lung cancer. JAMA Oncol 2016;2:46-54.

11. Le DT, Uram JN, Wang H, et al. PD-1 blockade in tumors with mismatchrepair deficiency. N Engl J Med 2015;372:2509-2520.

12. Liontos M, Anastasiou I, Bamias A, et al. DNA damage, tumor mutational load and their impact on immune responses against cancer. Ann Transl Med 2016;4:264.

13. Goodman AM, Kato S, Bazhenova L, et al. Tumor mutational burden as an independent predictor of response to immunotherapy in diverse cancers. Mol Cancer Ther 2017;16:2598-2608.

14. Snyder A, Makarov V, Merghoub T, et al. Genetic basis for clinical response to CTLA-4 blockade in melanoma. N Engl J Med 2014;371:2189-2199.

15. Rizvi H, Sanchez-Vega F, La K, et al. Molecular determinants of response to anti-programmed cell death (PD)-1 and anti-programmed deathligand 1 (PD-L1) blockade in patients with non-small-cell lung cancer profiled with targeted next-generation sequencing. J Clin Oncol 2018;36:633-641.

16. Salem ME, Xiu J, Weinberg BA, et al. Characterization of tumor mutation burden (TMB) in gastrointestinal (GI) cancers [abstract]. J Clin Oncol 2017; 35(Suppl):Abstract 530.

17. Jain A, Shroff RT, Zuo M, et al. Tumor mutational burden (TMB) and coexisting actionable mutations in biliary tract cancers (BTC) [abstract]. J Clin Oncol 2017;35(Suppl):Abstract 4086.

18. Chalmers ZR, Connelly CF, Fabrizio D, et al. Analysis of 100,000 human cancer genomes reveals the landscape of tumor mutational burden. Genome Med 2017;9:34
19. Hellmann MD, Ciuleanu TE, Pluzanski A, et al. Nivolumab plus ipilimumab in lung cancer with a high tumor mutational burden. N Engl J Med 2018; 378:2093-2104

20. Bartley AN, Luthra R, Saraiya DS, et al. Identification of cancer patients with Lynch syndrome: clinically significant discordances and problems in tissue-based mismatch repair testing. Cancer Prev Res (Phila) 2012;5: 320-327.

21. Cicek MS, Lindor NM, Gallinger S, et al. Quality assessment and correlation of microsatellite instability and immunohistochemical markers among population- and clinic-based colorectal tumors results from the Colon Cancer Family Registry. J Mol Diagn 2011;13:271-281.

22. Lee JH, Cragun D, Thompson Z, et al. Association between IHC and MSI testing to identify mismatch repair-deficient patients with ovarian cancer. Genet Test Mol Biomarkers 2014;18:229-235.

23. Vilkin $A$, Niv $Y$, Nagasaka $T$, et al. Microsatellite instability, MLH1 promoter methylation, and BRAF mutation analysis in sporadic colorectal cancers of different ethnic groups in Israel. Cancer 2009;115:760-769.

24. Gras E, Catasus L, Argüelles R, et al. Microsatellite instability, MLH-1 promoter hypermethylation, and frameshift mutations at coding mononucleotide repeat microsatellites in ovarian tumors. Cancer 2001;92:2829-2836.

25. Golan T, Raitses-Gurevich M, Kelley RK, et al. Overall survival and clinical characteristics of BRCA-associated cholangiocarcinoma: a multicenter retrospective study. Oncologist 2017;22:804-810.

26. Zou S, Li J, Zhou H, et al. Mutational landscape of intrahepatic cholangiocarcinoma. Nat Commun 2014;5:5696.

27. Birkbak NJ, Kochupurakkal B, Izarzugaza JM, et al. Tumor mutation burden forecasts outcome in ovarian cancer with BRCA1 or BRCA2 mutations. PLoS One 2013;8:e80023.

28. Strickland KC, Howitt BE, Shukla SA, et al. Association and prognostic significance of BRCA1/2-mutation status with neoantigen load, number of tumor-infiltrating lymphocytes and expression of PD1/PD-L1 in high grade serous ovarian cancer. Oncotarget 2016;7: 13587-13598.

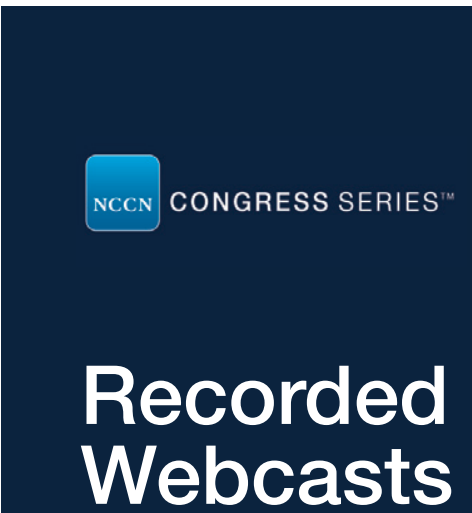

education.nccn.org/breast

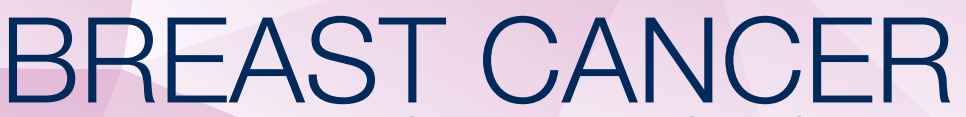

with Updates from the 2018 San Antonio Breast Cancer Symposium

Topics:

- Genetic/Familial High-Risk Evaluation in Patients with Breast Cancer

- Implementation and Implications of AJCC Cancer Staging Manual, $8^{\text {th }}$ Edition for Breast Cancer Screening

- Evidence-Based Integrative Therapies in Breast Cancer

- Utility of Multigene Assays in Management of Early Stage Breast Cancer

- Locoregional Treatment of Breast Cancer, Including SABCS Updates

- Management of Lymphedema after Breast Cancer Treatment

- Neoadjuvant and Adjuvant Therapies for Early Stage Breast Cancer, Including SABCS Updates

- New Systemic Therapies for Metastatic Breast Cancer, Including SABCS Updates 